\title{
A Delayed Wave of Death from Reproduction in Drosophila
}

\author{
Carla M. Sgrò and Linda Partridge*
}

\begin{abstract}
Mortality rates typically increase rapidly at the onset of aging but can decelerate at later ages. Reproduction increases the death rate in many organisms. To test the idea that a delayed impact of earlier reproduction contributes to both an increase in death rates and a later deceleration in mortality, the timing of the surplus mortality produced by an increased level of egg production was measured in female Drosophila. Reproduction produced a delayed wave of mortality, coincident with the sharp increase in death rates at the onset of aging and the subsequent deceleration of mortality. These results suggest that aging has evolved primarily because of the damaging effects of reproduction earlier in life, rather than because of mutations that have detrimental effects only at late ages.
\end{abstract}

A cost of reproduction is prevalent in animals and plants: Greater reproductive activity causes a decrease in life-span and in subsequent fecundity (1). Much less well understood is the time course of the impact of reproductive costs. Mortality rates in a population typically increase rapidly as aging occurs but have been seen to drop below this initial rate of increase at late, mainly postreproductive, ages in experimental animals and in humans (2). A delayed impact of earlier reproduction could produce both the high initial rate of increase in death rates and the deceleration of mortality rates. If the increase in death rates from reproduction is delayed and temporary, earlier reproduction would cause a subsequent wave of mortality, with an initial increase in mortality rates, followed by deceleration after the peak of the wave has passed.

Department of Biology, University College London, Wolfson House, 4 Stephenson Way, London NW1 2HE, UK.

*To whom correspondence should be addressed Email: l.partridge@ucl.ac.uk
One theory about the evolution of aging suggests that genes with beneficial effects early in life also have deleterious effects later on $(3,4)$. A delayed impact of early reproduction on later mortality rates would provide a general mechanism for this type of timedelayed pleiotropic gene action. Experimental manipulations of reproductive rate can have both immediate (5) and delayed (6) effects on mortality, but the timing of the effects of natural genetic variation for early fecundity has not been examined. We used artificially selected lines of the fruit fly Drosophila melanogaster and sterility induced by $\mathrm{x}$-ray irradiation or by a female-sterile mutant to test the hypotheses that (i) early reproduction causes a delayed wave of elevated mortality, and (ii) delayed effects of natural genetic variation for early fertility underlie the evolution of aging.

We used sets of five replicate artificially selected lines derived from a wild-type base stock of $D$. melanogaster. "Old" lines were selected by breeding adults at an old age, whereas "young" lines were propagated from young breeders. To contribute to future generations in the old lines, flies had to survive to the time of egg collection, and there was hence selection for increased adult survival. Adult life-span evolved to be greater in the old lines than in the young lines, and their fecundity in early life evolved to be lower, demonstrating a cost of reproduction. There were no significant differences in preadult development time, larval competitive ability, body size, or late adult fecundity between the lines from the two selection regimes (4). The difference in early fecundity between the young and old lines was apparent by day 7 and ceased by day $28(4,7)$. Egg production had ended by day 40 . In both experiments reported here, we first abolished the difference in reproductive rate between the young and the old lines to determine whether it was responsible for the difference in their death rates. We could then measure the timing of the impact of the differing levels of early reproduction in the young and old lines by comparing their death rates directly with each other and also with those of sterile flies. Egg production, exposure to males, and mating have all been shown to reduce survival in female D. melanogaster (8). We examined the impact of egg production on mortality trajectories in females, in either once-mated females or in virgins, in the absence of males.

In a first experiment, using a single cohort of flies, we compared the death rates of oncemated egg-laying females of the old and young lines with each other and with the death rates of females that were x-ray-irradiated as late pupae, which halts oogenesis (7, 9 ). In the x-irradiated females, the significant difference in mortality rates between the young and old lines was abolished (Fig. 1A) (10). The measured difference in late-age mortality rates between reproductive youngand the old-line females was therefore explained by the difference between them in early fecundity. The egg-laying young-line
Fig. 1. Mean of the natural logarithms of the age-specific mortality rates for the five replicate old and young selection line females when (A) irradiated as late pupae and (B) mated once and producing eggs. Solid circles and solid line, old selection lines; open circles and dashed line, young selection lines.

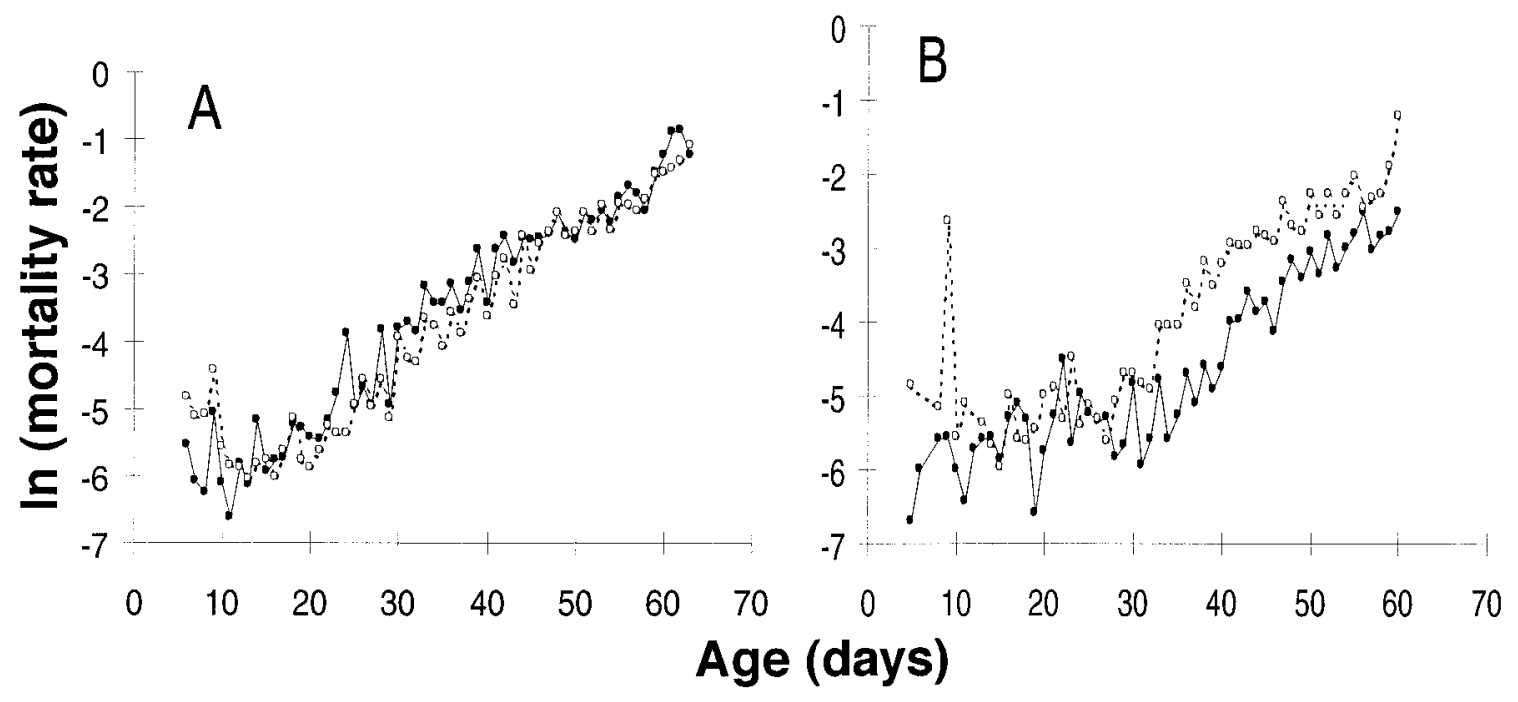




\section{R E P O R T S}

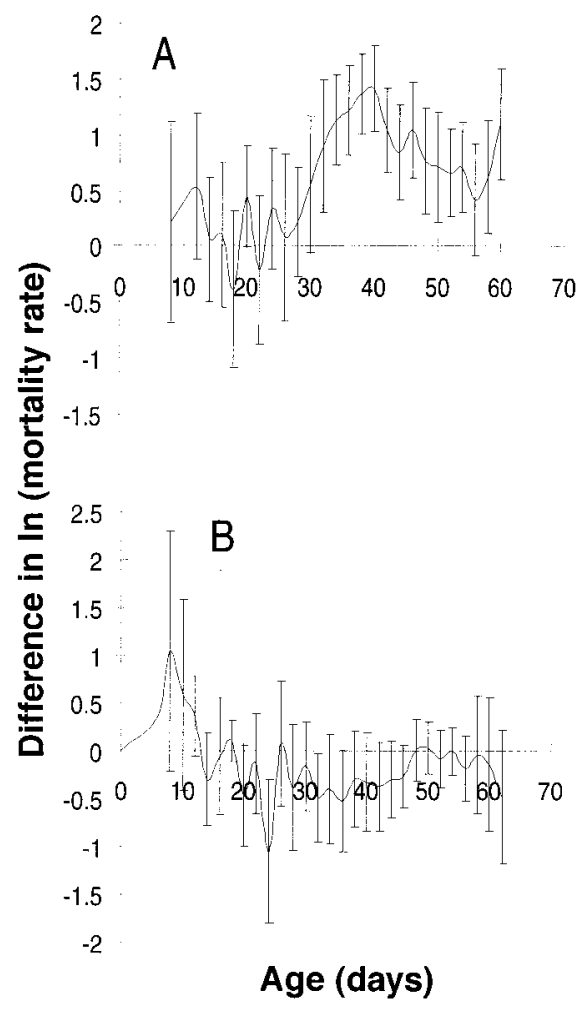

Fig. 2. Mean difference [and $95 \%$ confidence limits (CLs), shown by error bars] in the natural $\log$ of the mortality rates of egg-laying youngand old-line females (A) when mated once and producing eggs and (B) when irradiated as late pupae. Positive values indicate that young-line females had higher mortality rates. CLs were calculated from the five replicate differences between the matched pairs of old and young lines. The comparison was ended when the first young line went extinct.

females had significantly greater mortality rates after day 30 than did the old-line females, and the difference in death rates reached a peak at day 40 and thereafter tended to decline (Figs. 1B and 2A) (10); whereas in the irradiated females, differences in mortality rates were nonsignificant (Fig. 2B). The higher early egg production rate of the youngline females, which was over by day 28 (4,
7), therefore acted with a time delay to produce a wave of excess mortality later in their lives. X-rays would be expected to reduce mortality rates if their only effect was to induce sterility. However, $x$-irradiation significantly increased mortality rates in the old lines and had no consistent effect in the young lines (11). This finding suggested that $\mathrm{x}$-rays also caused somatic damage, and we confirmed this by $\mathrm{x}$-irradiation of females whose oogenesis was already blocked by the dominant mutant ovo ${ }^{D 1}$ (12). The full reduction in mortality caused by sterility was therefore not apparent in the x-irradiated females.

In a second experiment, we abolished egg production by a different method, which would be expected to have no direct effect on the adult soma, using the dominant ovo ${ }^{D I} \mathrm{mu}-$ tant. ovo encodes a zinc finger protein that is active only in the female germ line, and the mutant halts oogenesis at stage 4 (13). We hybridized males carrying the mutant, in the genetic background of the base stock from which the selection lines were derived, with each of the young and old selection lines and measured the mortality rates of the resulting $\mathrm{F}_{1}$ hybrid sterile females in single-sex groups. To produce genetically comparable egg-laying females, we hybridized each selection line to the base stock itself and examined the mortality rates of the resulting fertile hybrid virgin females in the same experiment as the sterile hybrid females (14). These two sets of females were therefore both genetically hybrids between the Dahomey base stock and each selection line, except for the presence of heterozygous $o v o^{D I}$ in the sterile females.

In the sterile females with ovo ${ }^{D 1}$ present, the significant difference in mortality rates between old and young lines was again abolished (15) (Figs. 3A and 4A), demonstrating that it was caused entirely by the difference in early egg production rate. $o v o^{D I}$ extended life span, and this extension was greater for the young- than for the old-line females, as would be predicted by the higher early fecundity of the former (16). In the egg-laying females, a wave of significantly increased mortality in the young-line females peaked at day 52, with nonsignificant differences in age-specific death rates until day 32 and after day 58 (Figs. 3B and 4B). Direct comparison of fertile and sterile females showed that the low early egg production rates of the fertile old-line hybrid females were not sufficient to elevate their subsequent death rates above those of the comparable sterile old-line hybrid females (Fig. 4C). In the young-line fertile hybrid females there was again evidence for a wave of increased mortality rates after the difference in reproductive rate had ceased (Fig. 4D). The low egg production of the old-line females at early ages may therefore have been insufficient to elevate their mortality to an extent that could be detected with these sample sizes.

The mortality rate deceleration, seen at late ages in other studies, was clearest in the present study in the egg-laying females illustrated in Fig. 3B, which lived the longest of the egg-laying groups. Deceleration was greatest in the young-line females and commenced at day 54 , when death rates started to drop. This coincided with the declining impact of earlier reproduction, which had peaked at day 52 (Fig. 4, B and D). Females of these selection lines become postreproductive by day 40. The wave of mortality consequent upon earlier egg production thus contributed to the mortality rate deceleration at postreproductive ages in these females.

The mechanisms by which reproduction acts with a time delay to increase mortality rates require further investigation. Reproduction may cause damage directly, and the effects may accumulate with time. Reproduction may also divert nutrients from repair and defense, resulting in more rapid accumulation of damage (17). The mortality rate deceleration at later ages could be explained as a consequence of the subsequent repair of damage from earlier ages, and of a diversion of resources to repair as reproduction wanes. Heterogeneity in frailty between the individuals in a population may also contribute to a declining impact of the cost of reproduction with time, if the survivors of the initial impact
Fig. 3. Mean natural logarithm of the agespecific mortality rates for (A) sterile females that were hybrids between each selection line and the Dahomey base stock males carrying $\mathrm{Ovo}^{\mathrm{D1}}$ and (B) fertile females that were hybrids between each selection line and the Dahomey base. Solid circles and solid line, old selection lines; open circles and dashed line, young selection lines.

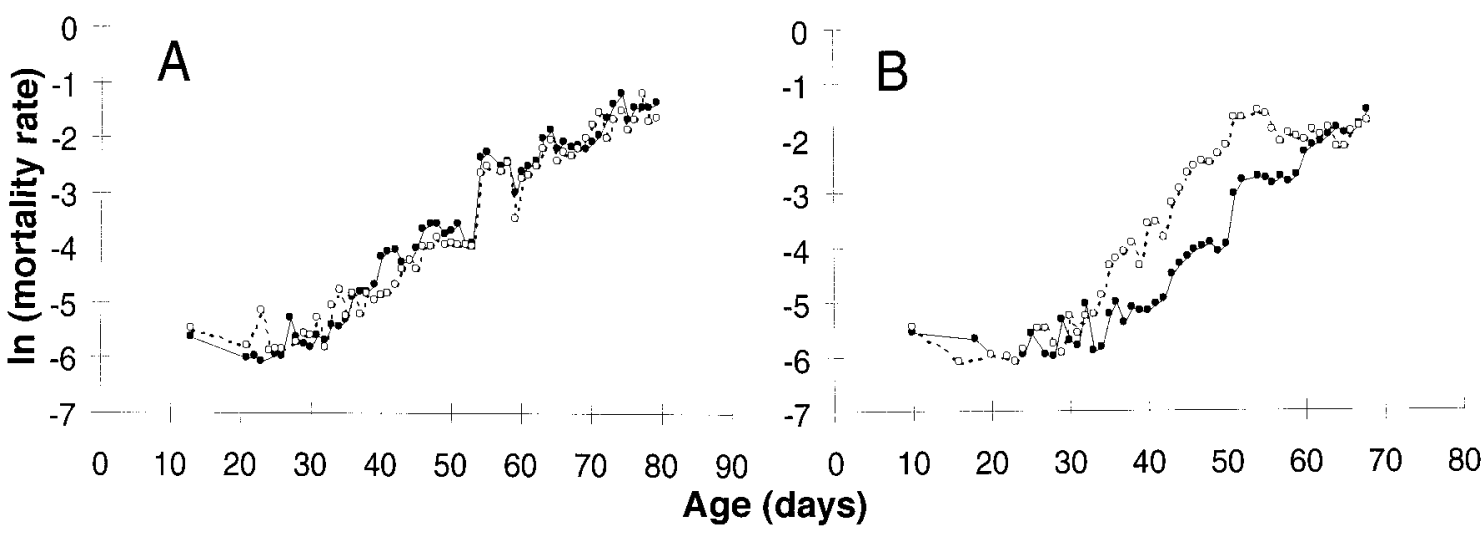

24 DECEMBER 1999 VOL 286 SCIENCE www.sciencemag.org 


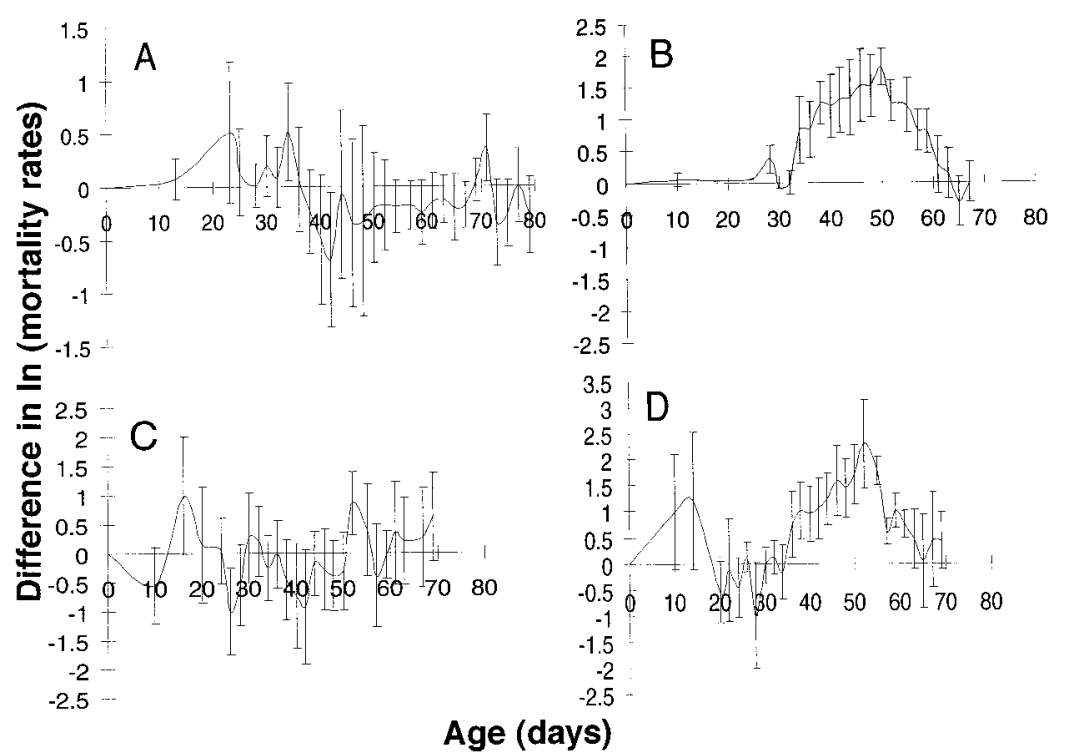

Fig. 4. Mean difference (and $95 \% \mathrm{CLs}$ ) in the natural logarithm of the age-specific mortality rates for (A) old and young sterile hybrid females, (B) old and young fertile hybrid females, (C) old-line fertile and sterile hybrid females, and (D) young-line fertile and sterile hybrid females. CLs were calculated from the five replicate differences between the matched pairs of old and young lines or between fertile and sterile hybrids.

of reproduction tend to be a more robust subset of their cohort (18).

The abolition of the difference in late-age mortality rates by sterility, whether induced by $\mathrm{x}$-irradiation or $o v o^{D I}$, has important implications for the evolution of aging. The young and old lines were the products of different naturally occurring genetic variants for the rate of aging because they were produced by artificial selection from a wild-type base stock. First, our data show that the lower death rate of the old lines is not a consequence of constitutive up-regulation of repair and defense processes (17), because this would be expected to persist when the difference in early egg production was abolished. Second, the results support the idea that aging evolves as a result of genes that have both beneficial effects at early ages and detrimental effects at late ages $(3,4)$. Furthermore, the results imply that the two effects are not the result of the effects of expression of these genes at different ages, but rather a direct impact of the early beneficial process that they produce (high egg production) on mortality at later ages. Finally, the results indicate that mutation accumulation causing late-age mortality (19) was not detectable in the young lines with these sample sizes, because any effects of mutation accumulation would be expected to persist after the removal of the difference in early reproductive rate. Mutations that specifically elevate death rates at late ages may therefore be rare (20).

\section{References and Notes}

1. G. C. Williams, Am. Nat. 100, 687 (1966); G. Bell and V. Koufopanou, Oxford Surv. Evol. Biol. 3, 83 (1986); D. Reznick, Oikos 44, 257 (1985).
2. A. Brooks, G. L. Lithgow, T. E. Johnson, Science 263 , 668 (1994); J. W. Curtsinger, H. H. Fukui, D. R. Townsend, J. W. Vaupel, Science 258, 461 (1992); J. R. Carey, P. Liedo, D. Orozco, J. W. Vaupel, Science 258, 457 (1992).

3. G. C. Williams, Evolution 11, 398 (1957); M. R. Rose and B. Charlesworth, Genetics 97, 187 (1981); M. R. Rose, Evolution 38, 1004 (1984); L. S. Luckinbill, R. Arking, M. J. Clare, W. C. Cirocco, S. A. Buck, Evolution 38, 996 (1984); B. J. Zwaan, R. Bijlsma, R. F. Hoekstra, Evolution 49, 649 (1995).

4. L. Partridge, N. Prowse, P. Pignatelli, Proc. R. Soc. London Ser. B. 266, 255 (1999).

5. L. Partridge and R. Andrews, J. Insect Physiol. 31, 393 (1985).

6. S. Trevitt, K. Fowler, L. Partridge, J. Insect. Physiol. 34, 821 (1988). M. Tatar, J. R. Carey, J. W. Vaupel, Evolution 47, 1302 (1993); N. Prowse and L. Partridge, J. Insect Physiol. 43, 501 (1997).

7. Flies were reared at low larval density ( 50 per vial), as during selection of the lines (4). Egg-laying females were obtained by mating virgin females from each selection line once with base stock males. To obtain sterile females, pupae were irradiated with $7.2 \mathrm{krad}$ at $451 \mathrm{rad} \mathrm{min}^{-1}$, which is the minimum dosage necessary to achieve full sterility. Egg-laying and sterile females were placed in cages at 300 to 400 females per cage and two replicate cages per selection line, under conditions that were the same as those used during selection. Deaths were counted daily. Early fecundity was measured by two 3-hour egg collecby two 6-hour egg collections on days 24 to 25 of adult life. There were no significant differences in fecundity (Student's $t$ test, $P>0.05$, for early and late fecundity) between replicate cages, and they were combined for further analysis. One-way analysis of variance (ANOVA), with replicate line nested within selection regime, showed that early fecundity was significantly higher in the young selection lines $(P<0.001)$, whereas old- and young-line females did not differ in late fecundity $(P>0.05)$. We compared the total mortality rates of the lines after day 28 , when fecundity was no longer greater in the youngline females. Young and old lines were assigned to matched pairs with the line numbers (1 through 5 ) arbitrarily allocated to them when artificial selection was initiated and which corresponded to experimental blocks. The Gompertz and the Weibull distributions on days 6 to 7 , and late fecundity was measured tions showed a significant lack of fit to the data. We therefore analyzed mortality rates, avoiding assumptions about the exact form of the increase in mortality rate with age. Mortality rates for the five matched pairs of young and old lines were compared after day 28 until the young line of each pair went extinct; comparisons were made with the distribution-free log tank test (10). For the egg-laying females, the mortality rates were significantly greater in the young lines in all five paired comparisons $(P<$ 0.0001 for four comparisons; $P=0.0031$ for the fifth). In the irradiated females, in four out of five pairs the young-line females had significantly (for three, $P<0.0001$; for one, $P<0.01$ ) lower mortality rates than did the old-line females; in the fifth, the difference was in the same direction but was nonsignificant (combined $P<0.0001$ ). The selection regimes did not differ in age-specific mortality rates. However, the total mortality rate after day 28 was significantly lower in the young lines, suggesting that they may have been more resistant to the damaging effects of $x$-rays $(10,11)$, or may have had higher levels of repair or response to stress.

8. J. Maynard Smith, J. Exp. Biol. 35, 832 (1958); M. J. Lamb, J. Insect Physiol. 10, 487 (1964); L. Partridge, A. Green, K. Fowler, J. Insect. Physiol. 33, 745 (1987); K. Fowler and L. Partridge, Nature 338, 760 (1989); L. Partridge and K. Fowler, J. Insect Physiol. 36, 419 (1990). T. Chapman, L. Liddle, J. M. Kalb, M. F. Wolfner, L. Partridge, Nature 373, 241 (1995).

9. M. Ashburner, Drosophila: A Laboratory Handbook (Cold Spring Harbor Laboratory Press, Cold Spring Harbor, NY, 1989).

10. R. G. Miller, Survival Analysis (Wiley, New York, 1981).

11. In every quartet, the post-day 28 mortality rate of the old replicate line was increased by irradiation $(P<0.0001$ for all five log rank comparisons). For the young lines, mortality was increased by irradiation in two pairs $(P<0.0001)$, irradiation did not significantly increase mortality in two $(P>0.05)$, and irradiation significantly lowered mortality in one $(P<$ 0.0001).

12. Virgin females of the Dahomey base stock from which the selection lines were derived were reared at low larval density. They were hybridized to males carrying the dominant mutant ${ } v o^{D 1}$ derived from two different stock origins and twice backcrossed into the Dahomey genetic background. Hybrid pupae were $x$-irradiated as in (12), and the subsequent survival of the irradiated females in single-sex groups of 400 in population cages was compared with that of unirradiated controls from the same cultures, using the log rank test. For females carrying the mutant from either stock of origin, irradiated females had significantly $(P<0.0001)$ higher mortality rates throughout life and after day 28 than did the unirradiated controls.

13. M. Mevel-Ninio, R. Terracol, F. C. Kafatos, EMBO J. 10 2259 (1991); B. Oliver, N. Perrimon, A. P. Mahowald, Genes Dev. 1, 913 (1987).

14. Virgin females of the selection lines were mated to either Dahomey males, to provide fertile hybrid females, or to males carrying ovo ${ }^{D}$ in the Dahomey genetic background, to provide sterile hybrid females. Hybrid females were reared at low larval density and were set up in population cages in single-sex groups of 300 to a cage per selection line hybrid. None of these females were ever mated.

15. For all five matched pairs of selection lines hybridized with the base stock, log rank tests showed that after day 28 , the young-line hybrid females had significantly $(P<0.0001)$ higher mortality rates than did the old-line females. For the hybrids carrying ovo ${ }^{D 1}$, log rank tests revealed that two pairs had nonsignificantly different mortality rates, in two pairs the young-line females had significantly $(P<0.01)$ lower mortality rates, and in one pair the old-line females had significantly $(P<0.05)$ lower mortality rates.

16. Life spans were normally distributed, and variances did not differ between groups. Two-way ANOVA of mean life span was used to test for the effects and interactions of sterility and selection regime. Replicate hybrid crosses were nested within selection. Life 
span was significantly greater in the old selection lines $(P<0.05)$ and when the females were made sterile with ovo ${ }^{D 1}(P<0.001)$. The response of the life span of young-line females to ovo ${ }^{D 1}$ was significantly $(P<0.01)$ greater than that of the old-line females.

17. T. B. L. Kirkwood, Nature 270, 301 (1977); and R. Holliday, Proc. R. Soc. London Ser. B. 205, 531 (1979).
18. J. W. Vaupel and A. I. Yashin, Am. Stat. 39, 176; J. W Vaupel et al., Science 280, 855 (1998).

19. P. B. Medawar, An Unsolved Problem in Biology (H. K. Lewis, London, 1952); B. Charlesworth, Evolution in Age-Structured Populations (Cambridge Univ. Press, Cambridge, ed. 2, 1994); D. Houle et al., Genetics 138, 773 (1994).

20. S. D. Pletcher, D. Houle, J. W. Curtsinger, Genetics 148, 287 (1998).
21. We thank T. Chapman for assistance with irradiation; T. Chapman, K. Fowler, and D. Gems for helpful discussion; and J. Carey, L. Harshman, P. Harvey, S. Pletcher, and J. Vaupel for comments on the manuscript. Supported by the Natural Environment Research Council (UK).

17 September 1999; accepted 24 November 1999

\title{
Linkage Disequilibrium and Recombination in Hominid Mitochondrial DNA
}

\author{
Philip Awadalla, ${ }^{1}$ Adam Eyre-Walker, ${ }^{2 *}$ John Maynard Smith ${ }^{2}$
}

\begin{abstract}
The assumption that human mitochondrial DNA is inherited from one parent only and therefore does not recombine is questionable. Linkage disequilibrium in human and chimpanzee mitochondrial DNA declines as a function of the distance between sites. This pattern can be attributed to one mechanism only: recombination.
\end{abstract}

For many years, it has been accepted that mitochondria are inherited exclusively from the mother in mammals and that their inheritance is therefore clonal. This assumption has been used extensively to date events in human prehistory, including the age of our last common female ancestor, "Eve," and the spread of Homo sapiens in Asia and Europe (1). However, mitochondria contain the enzymes necessary for homologous recombination (2), and there are at least two routes by which strict maternal inheritance of mitochondrial DNA (mtDNA) could be circumnavigated: (i) Paternal mitochondria enter the egg at fertilization $(3,4)$, and (ii) there are copies of mtDNA sequences in the nuclear genome (5) that could be transferred back to the mtDNA. Two reports have presented population genetic evidence of recombination in mtDNA $(6,7)$. Although doubts were raised about the quality of the data and the conclusions reached in one of these reports (8), subsequent analyses of newer data have reconfirmed the original result (9).

Recombination can also be detected by considering the relation between linkage disequilibrium (LD) and distance. As the distance between sites increases, the effect of recombination on LD should increase, whether recombination is a reciprocal or a nonreciprocal (gene conversion) process. Recombination should therefore manifest itself as a significant decline in LD with distance (1013).

\footnotetext{
${ }^{1}$ Institute of Cell, Animal and Population Biology, University of Edinburgh, Edinburgh EH9 1JT, UK. ${ }^{2}$ Centre for the Study of Evolution and School of Biological Sciences, University of Sussex, Brighton BN1 9QG, UK.

*To whom correspondence should be addressed. Email: a.c.eyre-walker@sussex.ac.uk
}

To measure disequilibrium, we used the Hill and Robertson measure (14)

$$
r^{2}=\frac{\left(p_{A B} p_{a b}-p_{a B} p_{A b}\right)^{2}}{p_{A}\left(1-p_{A}\right) p_{B}\left(1-p_{B}\right)}
$$

where $A$ and $a$ represent alleles at one locus, $B$ and $b$ represent alleles at the other locus, and $p_{X Y}$ is the frequency of the $X Y$ genotype. $r^{2}$ can range from 0 to 1 , from complete linkage equilibrium to complete disequilibrium, respectively. We only calculated LD values for pairs of sites at which both alleles were segregating at a frequency greater than $10 \%$ (in no case were more than two different nucleotides present at a site). There were two reasons for this restriction: (i) Alleles at higher frequencies tend to be older and therefore more likely to show evidence of past recombination events, and (ii) values of $r^{2}=1$ often occur by chance for alleles with low frequencies (15).

We analyzed both sequence and restriction fragment length polymorphism (RFLP) data for complete human mitochondrial genomes. To test whether LD declines with distance, we calculated Pearson's correlation coefficient, $\rho$, between LD and the distance between sites. To assess significance, we randomized the positions of sites and then recalculated the value of $\rho(11,13,16)$. This was repeated 5000 times; the significance is given as the proportion of random $\rho$ values that are more negative than the observed value. For the complete human mitochondrial sequences, we restricted analysis to synonymous variation, because selection can generate LD and there is no evidence of selection on synonymous codon usage in human mitochondrial sequences (7). In the RFLP data, it is generally not possible to determine whether the loss of a site is due to a synonymous or nonsynonymous change, so all variants in protein, tRNA, and ribosomal RNA coding sequences were analyzed. The human mitochondrial genome is a circular molecule 16,569 base pairs (bp) in length, so the longest distance between pairs is 8284 bp.

In humans, we have five independently collected data sets: 45 complete mtDNA sequences of diverse geographical origin (17) and four RFLP data sets spanning the whole genome, from 147 individuals from around the world (18), 86 Finnish and Swedish individuals (19), 153 Native Siberians (20), and 167 Native Americans (21). The relation between LD and the distance between sites, for four of these data sets, is shown in Fig. 1. In each case, there is an evident decline in LD with increasing distance, and in each case, the decline is significant. For example, Fig. 1A shows the 91 pairwise LD values for the 14 synonymous sites in the DNA sequence data set, plotted as a function of the distance between sites. The relation with distance is highly significant $(P=0.012)$. In the worldwide RFLP data set, there were only four sites segregating mutations at greater than $10 \%$; there is a negative correlation between LD and distance $(\rho=-0.365)$, but it is nonsignificant $(P=0.190)$. These patterns are consistent with those observed for nuclear sequences undergoing recombination (1013) and with population genetic predictions for the decay of $\mathrm{LD}$ due to recombination $(15,22,23)$.

For chimpanzees (Pan troglodytes), we used synonymous variants from the reduced form of nicotinamide adenine dinucleotide dehydrogenase subunit 2 (ND2) locus and data from the control region (CR) from 16 individuals of the subspecies verus from western Africa (24). There are about 3800 bp between the two sequences. There were 45 polymorphic sites and 990 pairwise comparisons. Again, there was a significant decline in linkage disequilibrium as distance between pairs of sites increased $(\rho=-0.147 ; P=0.0076)$. The proportion of pairs of sites with LD values significantly different from zero, at the $5 \%$ level, was much higher within the ND2 and CR sequences (166 out of 593 pairwise comparisons) than between them (46 out of 396) (Fisher's exact test, $P<10^{-6}$ ).

We have demonstrated that LD declines as a function of the distance between sites in humans and chimpanzees. This is expected if there is genetic recombination but hard to 